\title{
GPR prospecting in a layered medium via microwave tomography
}

\author{
Lorenzo Crocco and Francesco Soldovieri \\ Istituto per il Rilevamento Elettromagnetico dell'Ambiente (IREA), CNR, Napoli, Italy
}

\begin{abstract}
The tomographic approach appears to be a promising way to elaborate Ground Penetrating Radar (GPR) data in order to achieve quantitative information on the tested regions. In this paper, we apply a linearized tomographic approach to the reconstruction of dielectric objects embedded in a layered medium. The problem is tackled with reference to a two-dimensional geometry and scalar case when data are collected over a linear domain with finite extent. In particular, in order to increase the amount of independent available data, a multi-frequency/multi-view/ multi-static measurement configuration is considered. With reference to stepped-frequency radar, this means that for each working frequency and for each position of the transmitting antenna (moved along a linear domain), the electric field scattered by the buried targets is measured in several locations along the same linear domain. The proposed inversion approach is based on the Born approximation and a regularized solution is introduced by means of the Singular Value Decomposition (SVD). The problem of determining the optimal measurement configuration (in terms of number of frequencies and number of transmitting and receiving antennas) is also tackled by a numerical analysis relying on the Singular Value Decomposition (SVD). Numerical examples are provided to assess the effectiveness and robustness of the proposed approach against noise on data.
\end{abstract}

Key words GPR-microwave tomography-inverse scattering - ill-posed problems

\section{Introduction}

Ground Penetrating Radar (GPR) is widely employed in civil engineering, shallow subsurface prospecting applications and archaeology (Daniels, 1996) since it allows non invasive diagnostics of the probed domain in a fast and simple way.

GPR works by emitting a modulated electromagnetic pulse into the ground and by recording the strength of the echo produced by the interaction between the impinging waves and

Mailing address: Dr. Lorenzo Crocco, Istituto per il Rilevamento Elettromagnetico dell'Ambiente (IREA), CNR, Via Diocleziano 328, 80124 Napoli, Italy; e-mail: crocco.1.@irea.cnr.it the buried objects received at the air-soil interface in a monostatic or bistatic configuration. In the former case, the locations of the transmitting and the receiving antennas are coincident, whereas they are different in the latter case. By moving the antenna along a selected profile above the ground surface, a two-dimensional plot (radargram) is obtained in which the delay time of the recorded echoes (which can be related to the depth of the underground reflectors) is drawn versus the antenna position (Daniels, 1996).

This fast and simple operating mode is, however, able to survey only the presence and the location of the buried object but not its characteristics, unless additional a priori information is provided. Moreover, since it is based on the assumption that the velocity of the investigating wave is constant, it may often give misleading results. Finally, the interpretation of the radargrams is itself a difficult task which encompasses the ability and expertise of the user and requires the availability 
of a priori information about the domain under investigation.

A tomographic approach represents a possible way to overcome these limitations as it makes it possible to achieve quantitative reconstruction of the domain under investigation, in terms of location, shape and chemical/physical properties of the buried targets (Deming and Devaney, 1997). In this case, the problem at hand amounts to determining the dielectric and conductive properties of the probed domain starting from the knowledge of the electromagnetic field scattered under the incidence of known impinging fields. To this aim one has to perform the inversion of a pair of coupled integral equations which govern the electromagnetic scattering. Such an inversion is not a simple task (Colton and Kress, 1992) since it amounts to solving a non-linear ill-posed inverse problem. Indeed, due to the properties of the kernel of the integral equation relating the unknowns to the scattered field data (Bucci and Isernia, 1997; Pierri and Leone, 1999), in the presence of uncertainties on the data only a finite amount of independent information about the unknowns can be achieved. From a mathematical point of view, this means that a proper regularization strategy has to be adopted to restore the well-posedness of the problem (Bertero and Boccacci, 1998).

In addition, the nonlinear relationship between the data and the unknowns makes it very difficult to solve the problem, usually cast as the minimization of a non quadratic cost functional. Due to the large number of unknowns one usually has to deal with, stochastic optimization tools cannot be exploited and so deterministic minimization techniques have to be adopted (Isernia et al., 1997; Kleinman and van den Berg, 1993). Since convergence of deterministic approaches depends on the starting guess, the minimization can get stuck in a local minimum of the cost functional which actually represents a «false solution» of the problem (Isernia et al., 1997; Leone et al., 2001). The local minima problem leads to the necessity of making the number of the searched parameters as low as possible, or in an equivalent way to keep as high as possible the ratio between the amount of independent data and the number of the unknowns to be determined (Isernia et al., 2001). This requires availability of a priori information about the features of the unknowns and entails constraints on the extent of the probed domain.

Another class of solution algorithms is instead based on the adoption of linear approximations of the electromagnetic scattering (see, for instance, Deming and Devaney, 1997; Wang and Oristaglio, 2000; Pierri et al., 2001, 2002a). These linearized inversion algorithms require lower computational cost with respect to the nonlinear ones and no local minima problem arises for the corresponding functionals. The above factors make the linear inversion algorithms suitable in several applications concerning the diagnostics of large probed domain and allow real-time processing. Conversely, the adoption of approximate models of the electromagnetic scattering, introduces several limitations on the set of unknown functions which can be dealt with (Pierri et al., 2001).

In microwave tomography, the linearization is usually achieved by means of the Born Approximation (BA), which consists in approximating the total electric field inside the investigation domain with the incident one, i.e. the scattering object is a small perturbation with respect to the host medium. This assumption entails some constraints on the extent of the buried objects (which must be small with respect to the investigating wavelength) and on the difference between the dielectric properties of the objects and those of the background medium (which must be as low as possible) (Slaney et al., 1984).

In addition, also within the validity of the Born model, further constraints arise about the unknowns which can be dealt with. These limitations concern their allowable spatial variations and are due to the filtering effect of the relevant linear operator relating the unknowns to the scattered field data (Bucci et al., 2001a; Pierri et al., 2002a,b).

To this end, the Singular Value Decomposition (SVD) of the relevant linear compact operator (Bertero and Boccacci, 1998) provides a powerful tool to examine the amount of independent information carried by data, to discuss the class of retrievable unknowns, to determine the achievable spatial resolution limits 
in the retrieved tomographic images (Bucci et al., 2001a; Pierri et al., 2002a,b) and to give a stable solution of the problem.

In this paper, we apply the BA linear approach to the reconstruction of the spatial map of the dielectric and/or conductive properties of an investigation domain in the case of a layered medium. In particular, we consider a threelayered medium and inquire about the dielectric and conductive characteristics of an investigation domain completely embedded in the second layer. Such a scheme is representative of several realistic cases such as for instance masonry diagnostics and prospecting of the shallower layers of soil (Crocco et al., 2002). The twodimensional and scalar case is tackled with a filamentary excitation and the data are collected according to a multifrequency/multiview/multistatic strategy. As such, for each working frequency and for each position of the source, the data are measured in several locations along a linear domain of finite extent and very close to the first interface. The only a priori information about the problem regards the location and the extent of the investigation domain within which the objects are supposed to reside.

\section{The mathematical model}

The reference situation is shown in fig. 1 . The first region is homogeneous with relative dielectric permittivity $\varepsilon_{1}$ and conductivity $\sigma_{1}$. The second layer has extent equal to $d$ and is homogeneous with relative dielectric permittivity $\varepsilon_{2}$ and conductivity $\sigma_{2}$. The third region is homogenous with relative dielectric permittivity $\varepsilon_{3}$ and conductivity $\sigma_{3}$. The complex equivalent relative permittivity in each layer has an explicit dependency on frequency given by

$$
\varepsilon_{i}^{e q}(\omega)=\varepsilon_{i}-j \sigma_{i} / \omega \varepsilon_{0}
$$

$\varepsilon_{0}$ being the free space dielectric permittivity and $\omega$ the pulsation. The magnetic permeability is everywhere equal to that of the free-space, $\mu_{0}$.

We suppose that infinitely long cylindrical objects having cross sections invariant with respect to the $z$-axis are embedded in the second

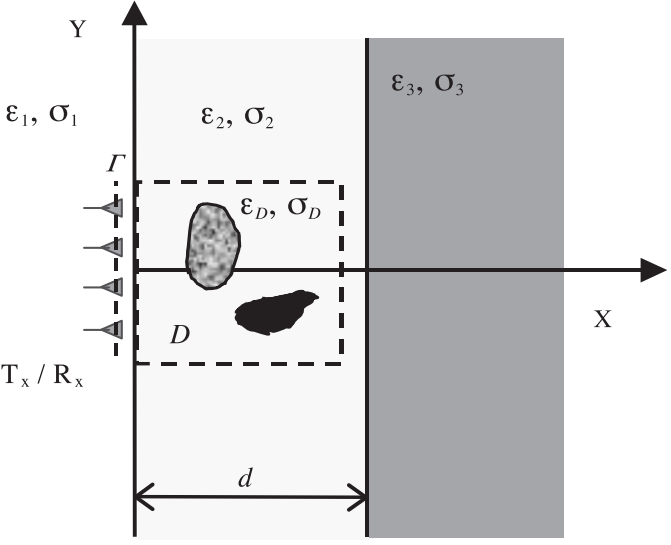

Fig. 1. Geometry of the problem.

layer and that their cross sections are contained within an a priori fixed square investigation domain $D$, completely embedded in the second layer. The unknowns of the problem are the spatial distribution of the relative dielectric permittivity and that of the conductivity over the investigation domain $D$.

The source of the incident field is a filamentary $z$-directed electric current (TM polarization), of non finite extent and constant along the $z$-axis, which radiates at different frequencies. Such a source is located in the first layer and is moved along the line $\Gamma$ parallel to the first interface and located very close to it. In order to collect multifrequency/multiview/multistatic data, for each working frequency and at each position of the source the electric field scattered by the buried targets is measured in several locations along $\Gamma$.

It is worth noting that such a set-up can be practically realized by using only one pair of antennas. As a matter of fact, exploiting a «synthetic array» strategy, one antenna acts as transmitter and the other one, which acts as receiver, is moved along the measurement line in the desired positions. By repeating this procedure for different locations of the transmitting antenna, one can achieve the desired set of data.

Under the above assumptions, the electromagnetic scattering phenomenon at hand is described by a two dimensional scalar model and 
it is governed by the following pair of integral equations (Chew, 1995)

$$
\begin{aligned}
& E\left(\boldsymbol{r}, \boldsymbol{r}_{t}, \omega\right)-E_{\mathrm{inc}}\left(\boldsymbol{r}, \boldsymbol{r}_{t}, \omega\right)=k_{2}^{2} \int_{D} E\left(\boldsymbol{r}^{\prime}, \boldsymbol{r}_{t}, \omega\right) \\
& \cdot \chi\left(\boldsymbol{r}^{\prime}, \omega\right) g_{22}\left(\boldsymbol{r}, \boldsymbol{r}^{\prime}, \omega\right) d \boldsymbol{r}^{\prime} \boldsymbol{r} \in D, \boldsymbol{r}_{\boldsymbol{t}} \in \Gamma \\
& E_{s}\left(\boldsymbol{r}_{r}, \boldsymbol{r}_{t}, \omega\right)=k_{2}^{2} \int_{D} E\left(\boldsymbol{r}^{\prime}, \boldsymbol{r}_{t}, \omega\right) \chi\left(\boldsymbol{r}^{\prime}, \omega\right) . \\
& g_{21}\left(\boldsymbol{r}_{r}, \boldsymbol{r}^{\prime}, \omega\right) d \boldsymbol{r}^{\prime} \boldsymbol{r}_{r}, \boldsymbol{r}_{t} \in \Gamma
\end{aligned}
$$

where the time-factor $\exp (j \omega t)$ has been omitted, $k_{2}$ is the (complex) wavenumber in the second medium, $E_{\text {inc }}, E$ and $E_{S}$ are the incident field in $D$ (i.e. the field in the absence of the object), the total field induced inside the investigation domain $D$ and the scattered field on $\Gamma$, respectively; $\boldsymbol{r}, \boldsymbol{r}_{t}$, $\boldsymbol{r}_{r}$ denote the generic point in the domain $D$, the generic location of the source and the generic measurement point, respectively.

The quantities $g_{21}$ and $g_{22}$ are the SommerfeldGreen's functions pertaining to the considered geometry and their expressions, as well as that of the incident field are reported in Appendix.

The contrast function $\chi$ relates the searched (complex) permittivity (defined in the domain $D$ ) to that of the background (medium 2) and is defined as

$$
\chi(\omega, \boldsymbol{r})=\frac{\varepsilon_{D}^{e q}(\omega, \boldsymbol{r})}{\varepsilon_{2}^{e q}(\omega)}-1
$$

where

$$
\varepsilon_{D}^{e q}(\omega, \boldsymbol{r})=\varepsilon_{D}(\boldsymbol{r})-j \sigma_{D}(\boldsymbol{r}) /\left(\omega \varepsilon_{0}\right) .
$$

Such a complex function changes with frequency and this does not allow to consider it as a convenient unknown in the multifrequency case. However, assuming a priori that $\varepsilon_{i}$ and $\sigma$ in eq. (2.1) as well as $\varepsilon_{D}$ and $\sigma_{D}$ in eq. (2.4) are independent of frequency, we can introduce a pair of frequency independent real functions

$$
\Delta \varepsilon=\varepsilon_{D}-\varepsilon_{2}, \Delta \sigma=\frac{\sigma_{D}-\sigma_{2}}{\omega_{m} \varepsilon_{0}}
$$

$\omega_{\text {m }}$ being the maximum working frequency. These functions are simply related to $\chi$

$$
\chi(\omega, \boldsymbol{r})=\frac{1}{\varepsilon_{2}^{e q}}\left(\Delta \varepsilon(\boldsymbol{r})-\frac{j \omega_{m}}{\omega} \Delta \sigma(\boldsymbol{r})\right)
$$

and in what follows we will assume them as the actual unknowns of our inverse problem. By so doing we are able to deal with frequency independent unknowns, $\Delta \varepsilon$ and $\Delta \sigma$, without neglecting the dispersive nature of the problem.

\section{The linearized tomographic approach}

In its general formulation, see eqs. (2.2a,b), the problem we are dealing with is non-linear. However, in some cases it is possible to describe the problem by means of an approximated model.

The Born approximation (Slaney et al.,1984) consists in neglecting the mutual interactions between the objects and amounts to assuming the internal field $E$ in $D$ equal to the unperturbed one. This allows a considerable simplification since the electromagnetic scattering is modeled by means of a single linear integral equation.

When the targets are embedded in a lossy medium, a large number of cases can be modeled by means of the BA (Bucci et al., 2001b), since the presence of losses smoothes multiple scattering effects. Considering that in masonry diagnostics as well as in subsurface prospecting involved media are usually lossy, we will consider the BA in the following.

Accordingly, the mathematical model is given by

$$
\begin{gathered}
E_{s}\left(\boldsymbol{r}_{r}, \boldsymbol{r}_{t}, \omega\right)=k_{2}^{2} \int_{D} \frac{E_{\mathrm{inc}}\left(\boldsymbol{r}^{\prime}, \boldsymbol{r}_{t}, \omega\right) g_{21}\left(\boldsymbol{r}_{r}, \boldsymbol{r}^{\prime}, \omega\right)}{\varepsilon_{2}-j \sigma_{2} / \omega \varepsilon_{0}} \times \\
\quad \times\left(\Delta \varepsilon\left(\boldsymbol{r}^{\prime}\right)-\frac{j \omega_{m}}{\omega} \Delta \sigma\left(\boldsymbol{r}^{\prime}\right)\right) d \boldsymbol{r}^{\prime}
\end{gathered}
$$

and the problem can be cast as the inversion of the linear operator given by eq. (3.1) and 
defined as

$\mathcal{L}_{\mathrm{DB}}: x \in X \subset L^{2}(D) \times L^{2}(D) \rightarrow E_{s} \in Y \subset L^{2}(\Gamma \times \Gamma \times \Phi)$.

In eq. (3.2) $X$ is a subspace containing all the pairs of functions $x=(\Delta \varepsilon, \Delta \sigma)$ and the data space $Y$ is a subspace containing the scattered fields corresponding to all possible measurement positions over $\Gamma$, all possible source positions over $\Gamma$ and to the frequency band $\Phi$.

Due to the properties of its kernel, $\mathcal{L}_{\mathrm{DB}}$ is a compact operator, so that its inversion is an ill-posed problem and the necessity of restoring well-posedness arises (Bertero and Boccacci, 1998). Therefore a suitable regularization scheme has to be adopted to set up a stable and reliable solution.

A convenient tool to achieve a regularized inversion is provided by the SVD of the operator $\mathcal{L}_{\mathrm{DB}}$, which defines a triplet $\left\{\sigma_{n}, u_{n}, v_{n}\right\}_{n=0}^{\infty}$ such that

$$
\mathcal{L}_{\mathrm{DB}}\left(u_{n}\right)=\sigma_{n} v_{n}, \quad \mathcal{L}_{\mathrm{DB}}{ }^{+}\left(v_{n}\right)=\sigma_{n} u_{n}
$$

wherein $\mathcal{L}_{\mathrm{DB}}{ }^{+}$is the adjoint operator of $\mathcal{L}_{\mathrm{DB}}$, $\left\{\sigma_{n}\right\}_{n=0}^{\infty}$ denotes the sequence of the singular values ordered in non increasing sequence and $u_{n}$ and $v_{n}$ form the basis for the space of the visible objects (that is the objects which can be recovered by the error-free data) and for the closure of the range (that is the space of the noise-free data) of the operator, respectively.

Exploiting the SVD of the relevant operator, the formal solution of integral eq. (3.1) can be cast as

$$
x=\sum_{n=0}^{\infty} \frac{1}{\sigma_{n}}<E_{s}, v_{n}>u_{n}
$$

where <.,.> denotes the scalar product in the data space.

The compactness of $\mathcal{L}_{\mathrm{DB}}$ entails that the sequence of the singular values asymptotically decays to zero (Bertero and Boccacci, 1998). Therefore the ill-posedness of the inverse problem given by eq. (3.1) immediately appears: indeed, in the presence of noise on data, the contributions to the solution related to the singular values closer to zero will be strongly corrupted by noise and the result of the inversion will become unstable.

A simple way to tackle such an instability consists in truncating the expansion (3.4) to those terms that are not overwhelmed by noise. Therefore we assume as a regularized solution of our problem the one obtained by means of the truncated SVD expansion

$$
x=\sum_{n=0}^{N} \frac{1}{\sigma_{n}}<E_{s}, v_{n}>u_{n} .
$$

By restricting the solution space to that spanned by the first $N+1$ singular functions, this regularized solution is stable with respect to errors on data. However, since it is achieved by considering only a reduced number of terms in eq. (3.4), this procedure will lead to an approximate representation of the sought unknown. Therefore, the choice of the index $N$ represents a trade-off between the accuracy of the solution and its stability with respect to noise. Such an optimal choice, in its turn, is actually related to the estimated level of noise and to the model error (*). As a consequence, the optimal choice may be a chimera, because the model error depends on the unknown scattering object. Lacking definite criteria to determine such an optimal choice, we have chosen in our regularized approach to reject the contributions related to singular values $20 \mathrm{~dB}$ lower than the maximum one.

\subsection{The discretization procedure}

The numerical implementation of the inversion procedure described above is based on an equivalent matrix formulation of the problem in which only real vectors are involved.

As a first step, one has to discretize the unknown parameters within the investigation domain $D$. In order to satisfy the criteria outlined in Richmond (1965), the pair of unknown functions is expanded by using as a basis the pulse functions defined within

(*) The model error is the discrepancy between the actual scattered field data and those that would result according to the BA. 
$N \times N$ square cells whose side is $\lambda / 20$ long, $\lambda$ being the minimum wavelength, thus rendering the results of the discretized version of the problem very close to those of the continuous problem in eq. (3.1). The vector of the unknowns is now defined as a column vector of dimension $2 N_{c}^{2}$, whose first $N_{c}^{2}$ rows contain $\Delta \varepsilon$, and the second $N_{c}^{2}$ rows contain $\Delta \sigma$.

A discrete set of measurements $N_{m}$, incident fields $N_{v}$ and frequencies $N_{f}$ is considered, leading to $M=N_{m} \times N_{v} \times N_{f}$ complex data. Therefore, the data vector is defined as a column vector of dimension $2 M$, in which the real part of the scattered fields (considered at each frequency, incident field and measurement point) is stored in the first $M$ rows, and the imaginary part is stored in the latter $M$ rows.

By following this procedure one achieves the matrix equation:

$$
\left[\begin{array}{ll}
\boldsymbol{A} & \boldsymbol{B} \\
\boldsymbol{C} & \boldsymbol{D}
\end{array}\right] \cdot\left[\begin{array}{c}
\Delta \varepsilon \\
\Delta \sigma
\end{array}\right]=\left[\begin{array}{l}
\operatorname{Re}\left(E_{S}\right) \\
\operatorname{Im}\left(E_{S}\right)
\end{array}\right]
$$

wherein $\boldsymbol{A}, \boldsymbol{B}, \boldsymbol{C}, \boldsymbol{D}$ are block matrices of dimension $M \times N_{c}^{2}$, computed by separating the discretized counterpart of eq. (3.1) in its real and imaginary parts.

The discretized problem, as stated in eq. (3.6), can be now solved by means of the numerical SVD applied to real matrices.

\section{Fixing the measurement set-up via SVD}

Besides defining the regularized solution of the linear inversion at hand, the SVD can be exploited as a tool to fix the number of antennas of the measurement set-up and to investigate the effect of using a different number of frequencies in a given band.

This can be performed by examining the effect of the change in the number and locations of the transmitting and receiving antennas on the number of the singular values $N$ retained in the summation (3.5). According to the choice of the above section, we assume $N$ as the number of singular values larger than 0.1 times the maximum singular value.
The analysis is performed under the simplifying hypothesis of transmitting and receiving antennas uniformly spaced over the observation domain. In addition, thanks to the arguments in Bucci et al. (2001a), it is convenient to assume that the number of transmitter and receiver locations is the same. In this way it results $N_{v}=N_{m}$, that is for each of the $N_{m}$ positions of the transmitter, $N_{m}$ measurement are collected (multiview/multistatic configuration).

One can expect that when the number of transmitters and receivers increases, some independent information about the unknown is added and so the number $N$ of useful singular values to be retained in (3.5) increases. However, since the available information carried by the scattered fields is finite, it is expected that a maximum number of antennas exists beyond which the addition of further antennas does not change in a significant way the number of useful singular values to be employed in the reconstruction.

This is a very important point as in any practical application an increase in the complexity of the set-up means increasing costs in terms of measurement time and computational burden.

To give an example of how the SVD can be usefully exploited to this aim, we have considered a case typical of masonry diagnostics, in which media 1 and 3 are the free space and medium 2 is $d=2 \mathrm{~m}$ thick and has a relative dielectric permittivity of 6 and a conductivity of $1 \times 10^{-3} \mathrm{~S} / \mathrm{m}$. The investigated domain is $1 \mathrm{~m} \times 1 \mathrm{~m}$ starting from the first interface and the receivers and transmitters are placed on a line $\Gamma$ $1 \mathrm{~m}$ long placed just above the interface.

The following analysis concerns only the relevant linear operator and therefore it does not depend on the targets, but only on the geometrical characteristics of the investigated domain, the background permittivity and the measurement configuration.

Firstly, let us consider a single frequency case at $300 \mathrm{MHz}$ and determine which is the suitable number of antennas. This is performed by evaluating the SVD of the matrix defined in eq. (3.6) when changing the number of transmitters and receivers. Figure 2 shows the behavior of the normalized singular values greater than the threshold (assumed equal to $-20 \mathrm{~dB}$ ) in these various situations. As can be seen, the number of 




Fig. 2. Behavior of the normalized singular values of $\mathcal{L}_{\mathrm{DB}}$ at $300 \mathrm{MHz}$ when changing the number of transmitter (and receiver) positions. A suitable choice is $N_{m}=9\left(N_{v}=9\right)$.

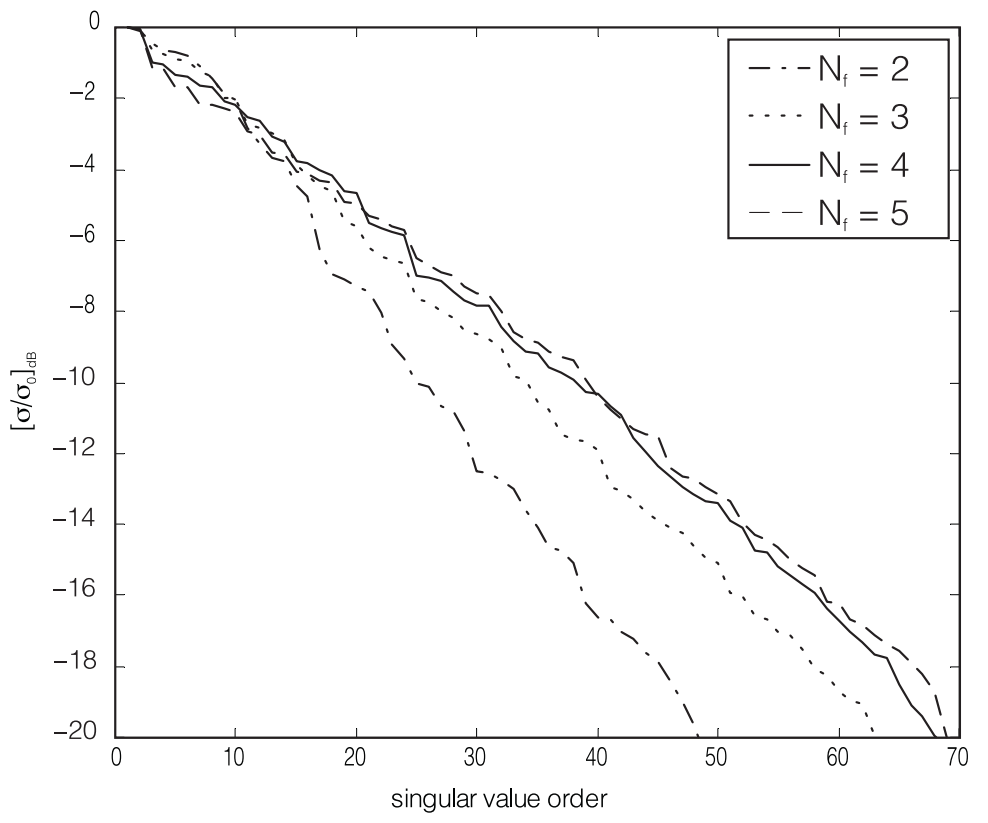

Fig. 3. Behavior of the singular values of $\mathcal{L}_{\mathrm{DB}}$ when varying the number of frequency steps in the $100-300 \mathrm{MHz}$ band: $N_{f}=2(100,300)$, dashed-dotted line; $N_{f}=3(100,200,300)$, dotted line; $N_{f}=4(100,150,225,300)$, solid line; $N_{f}=5(100,150,200,250,300)$, dashed line. 
useful singular values remains nearly unchanged when varying $N_{m}$ from 9 to 13 or more, while it is considerably lower when considering $N_{m}$ equal to 5 or less. On the basis of such a simple analysis we can then conclude that a suitable choice for $N_{m}$ (and hence for $N_{v}$ ) is 9 .

Secondly, by assuming such a number of transmitter and receiver locations, we can then investigate the effect of the addition of the working frequencies in the band $100-300 \mathrm{MHz}$. Figure 3 shows behavior of the singular values when increasing the number of frequencies in the 100-300 MHz band. First of all, it appears than increasing such a number has beneficial effects on the number of singular values. However, it also appears that considering more than four frequencies does not bring a definite advantage. In what follows we will assume that the data are collected at 100, 200 and 300 $\mathrm{MHz}$. Although a (slightly) larger number of singular values would be achieved considering four frequencies, this choice satisfies the need of keeping the computational burden as low as possible, without dramatically reducing the number of singular values.

In conclusion, we have shown that exploiting the SVD tool it is possible to simply determine the measurement set-up, both in terms of the number of antennas and in terms of the number of frequencies to consider.

\section{Numerical examples}

In this section we will give some examples aimed to show the reconstruction capabilities of the proposed inversion approach. The reference situation is the one depicted in fig. 1. The measurement set-up is designed according to the criteria outlined in the previous section and considers three equispaced frequencies in the band 100-300 MHz. At each frequency, in correspondence of each of the nine positions of the transmitting antenna, the scattered field is measured at nine equispaced positions along the measurement line $\Gamma, 1 \mathrm{~m}$ long and placed very close to the first interface. The investigated domain is $1 \mathrm{~m} \times 1 \mathrm{~m}$ wide, starting from the first interface and is embedded in a layer which is $d=2 \mathrm{~m}$ thick. The dielectric and conductive properties of the three media are the same as in the previous section.

In all the examples, the data have been synthetically generated by means of a forward solver which exactly solves eq. (2.2a,b) and then corrupted by means of an additive white noise to simulate measurement error.

The aim of these examples is twofold. First, we want to show that the proposed approach can furnish qualitative information on the investigated region even when the model error is very large. As a matter of fact, unless a very low model error is present, it is not possible to achieve a quantitative reconstruction (even in the noiseless case) due to the discrepancy between the actual (synthetically generated) data and the data that would arise from the BA model. Second, we want to show how the proposed regularization is a robust inversion tool when considering noise corrupted data. Therefore, we will show for each case the reconstruction achieved in the (ideal) case of clean data and that obtained when data are corrupted by noise.

In the first example, we consider the problem of imaging a rectangular void. Due to the strong difference between the permittivity of the target and that of the background, the model error is quite large (about 100\%), and we do not expect to achieve a quantitative reconstruction. Figure 4a shows a comparison between the modulus of the (simulated) scattered data at $200 \mathrm{MHz}$ and the corresponding field that would result according to the BA: as it can be seen the difference between the two fields is very large. Figure $4 b$ shows the comparison between the clean data and the data corrupted with an additive Gaussian noise with an SNR of $10 \mathrm{~dB}$, which does not change at the various frequencies. By comparing fig. $4 \mathrm{a}$, b one can observe that in this case the larger error comes from the failure of the BA model.

Several factors affect the model error. Among them the difference between the permittivity of the target and that of the background plays a major role. In table I one can observe how the model error varies when changing the permittivity of the target (the rectangular void we are considering): the void certainly represents a situation in which the BA assumptions are strongly violated.

Another interesting point is the behavior of the model error as a function of the working 


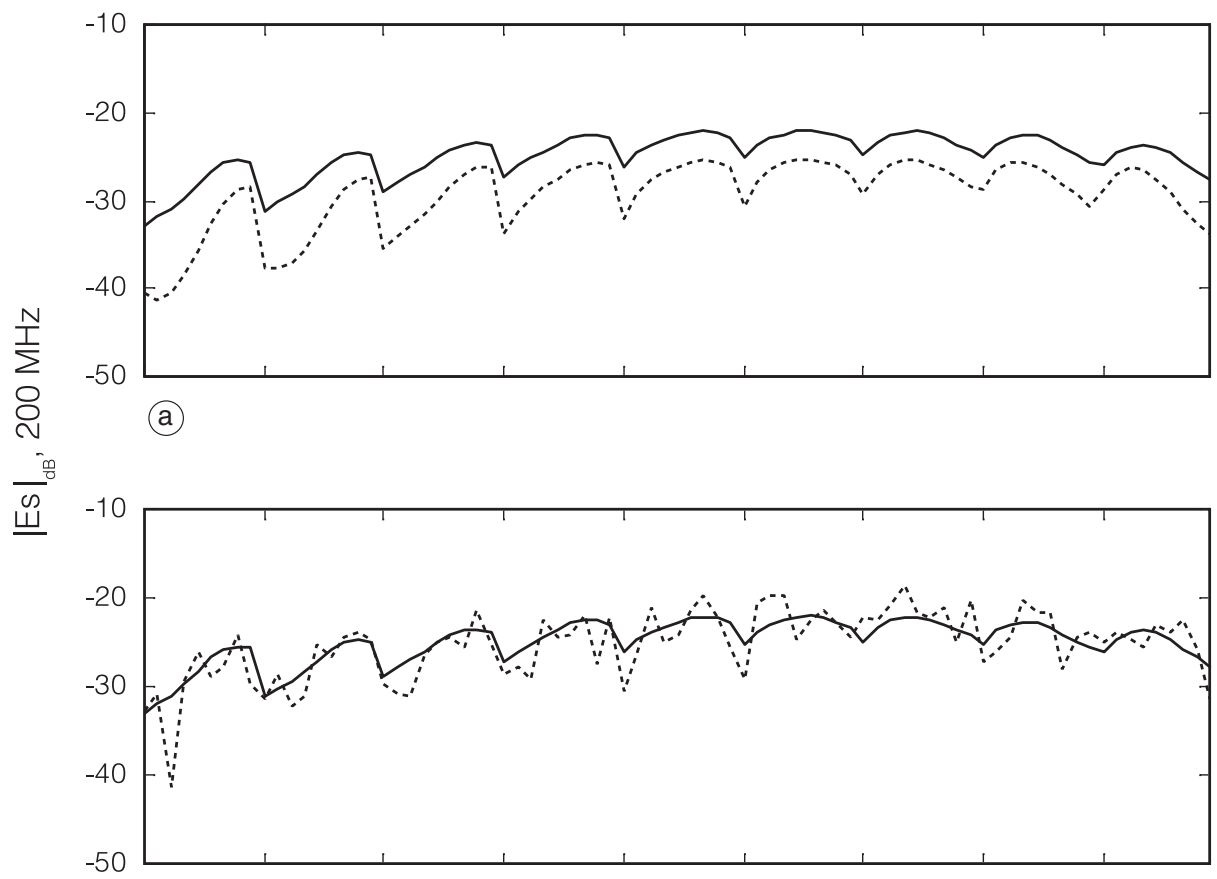

(b)

Fig. 4a,b. Case 1: a rectangular void. a) Comparison between actual scattered data (solid line) and data that would result according to the BA model (dotted line). b) Comparison between clean data (solid line) and data corrupted by noise with an SNR $=10 \mathrm{~dB}$ (dotted line). The plotted quantity is the modulus of the field at $200 \mathrm{MHz}$ for each location of the transmitter and the receiver.

Table I. Behavior of the model error when changing the value of the target permittivity. The larger the relative difference between the background permittivity $\left(\varepsilon_{b}=6\right)$ and the target one the larger the model error is.

\begin{tabular}{cc}
\hline \hline Target permittivity & Model error $\%$ \\
\hline 1 & 116 \\
2 & 115 \\
3 & 100 \\
4 & 69 \\
5 & 33 \\
5.9 & 3 \\
7 & 31 \\
8 & 59 \\
9 & 80 \\
10 & 94
\end{tabular}

frequency. In the case of an empty cavity, we can observe that the model error decreases with frequency, being equal to $118 \%$ at $300 \mathrm{MHz}$, $96 \%$ at $200 \mathrm{MHz}$ and $52 \%$ at $100 \mathrm{MHz}$. This can be explained observing that when decreasing the working frequency, the corresponding wavelength increases and therefore the electrical dimension of the target decreases too. This entails that, as long as the working frequency decreases, the validity of BA is ensured.

However, when decreasing the working frequency the amount of independent information carried by data decreases (Pierri et al., 2001, 2002a) and therefore only an approximation of the sought unknown with a lower resolution can be achieved (Pierri et al., 2002b). This is the main reason why frequency diversity is very 
important in microwave tomography approaches. As a matter of fact, exploiting multifrequency data it is possible to jointly consider data affected by a low model error (those pertaining to the lower frequency) and data which carry «more» independent information (those pertaining to the higher frequency).

In order to show the effect of the model error on the reconstruction capabilities of our approach, let us first consider the case of clean data. Figure 5 plots the modulus of the retrieved contrast (evaluated at $200 \mathrm{MHz}$ placing the retrieved $\Delta \varepsilon$ and $\Delta \sigma$ in eq. (2.6). As expected, we cannot achieve a quantitative reconstruction, nevertheless the regularized inversion approach locates the target properly (but for a small misplacement). The effect of the model error is also evident as a «ghost» appears in the deeper part of the region. This is related to the mutual interactions between the target and the second interface which are indeed neglected under the BA.

In order to prove the stability of our approach against noise on data, we repeated the inversion considering noise corrupted data and the modulus of the reconstructed contrast function is reported in fig. 6 . As can be seen, the achieved reconstruction is almost unchanged compared to the clean data case, apart for an obvious (small) enhancement of the artifacts. The inversion procedure is quite fast as it takes only a couple of minutes to run on a $200 \mathrm{MHz}$ Pentium II computer.

In the second example, we consider the case of imaging a conductivity anomaly; the target has the same permittivity as the background but a slightly different conductivity $\left(9 \times 10^{-3}\right.$ $\mathrm{S} / \mathrm{m})$. While in the previous example the main (theoretical) contribution to the scattering data was due to $\Delta \varepsilon$, in this case the main contribution is due to $\Delta \sigma$. Due to the small difference between the conductivity of the target and that of the background, a lower model error is expected, which indeed is about $13 \%$ (and is almost constant with frequency). This entails that the actual data and the data arising from the BA model are very similar; as pointed out by the comparison between the two fields at 200 $\mathrm{MHz}$ which is given in fig.7a. On the other hand, when comparing clean and noise corrupted data

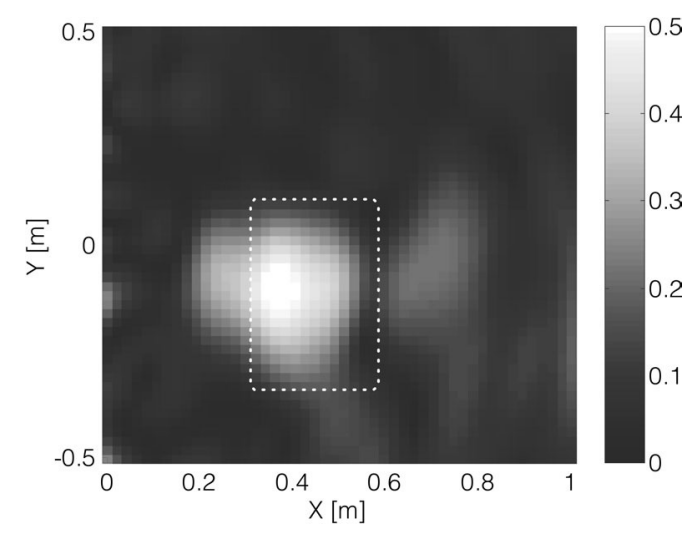

Fig. 5. Case 1: a rectangular void. Modulus of the reconstructed contrast when considering clean data. The reference shape is reported as a contour plot.



Fig. 6. Case 1: a rectangular void. Modulus of the reconstructed contrast when considering noise-corrupted data $(\mathrm{SNR}=10 \mathrm{~dB})$.

(fig. 7b), it appears that in this case the effect of measurement error is definitely more relevant than in the previous case. Considering the clean data first, the modulus of the reconstructed contrast is plotted in fig. 8. The target can be clearly located and also the quantitative value of the contrast is determined with a good accuracy (the actual value of the modulus of the contrast would be 0.08).

Also in this case we repeated the test by corrupting the data with an additive white noise $(\mathrm{SNR}=10 \mathrm{~dB})$ and again the achieved result, see 


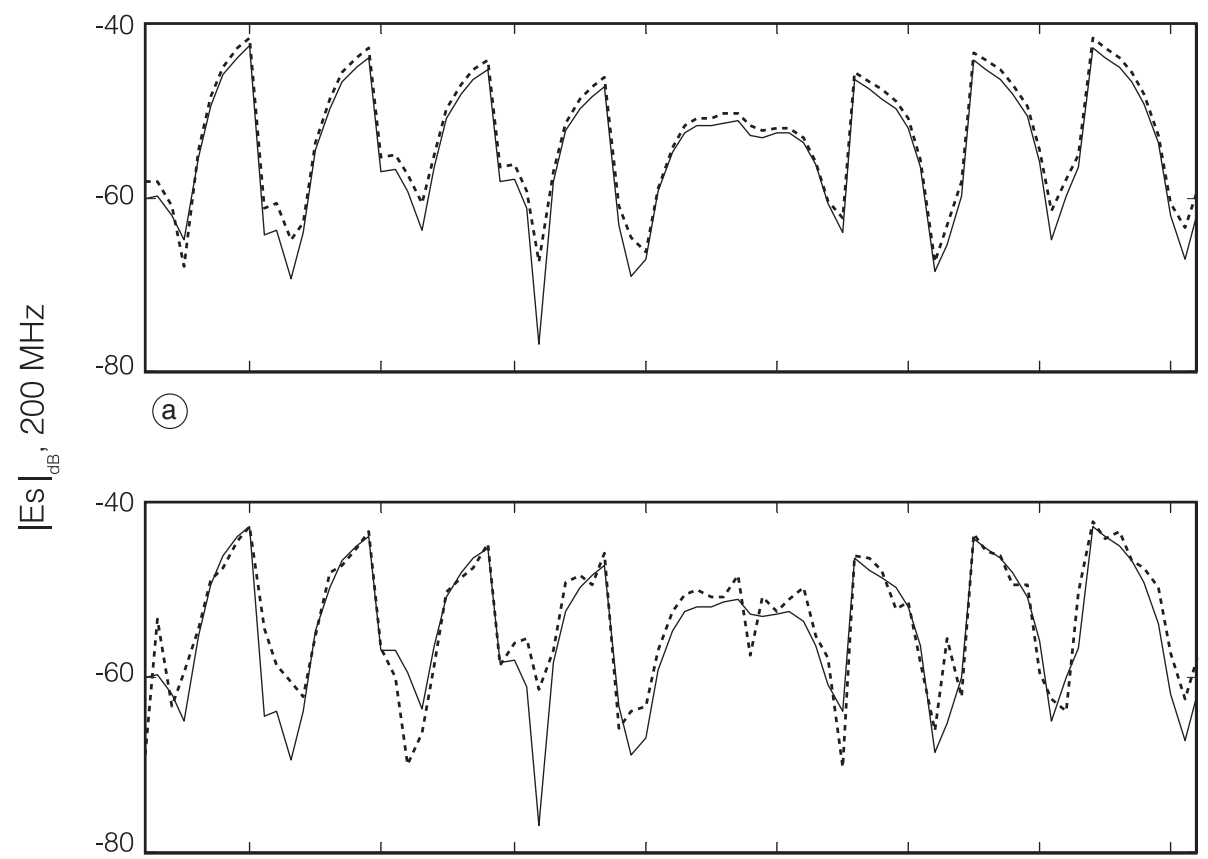

(b)

Fig. 7a,b. Case 2: a conductivity anomaly. a) Comparison between actual scattered data (solid line) and data that would result according to the BA model (dotted line). b) Comparison between clean data (solid line) and data corrupted by noise with an SNR $=10 \mathrm{~dB}$ (dotted line). The plotted quantity is the modulus of the field at $200 \mathrm{MHz}$ for each location of the transmitter and the receiver.

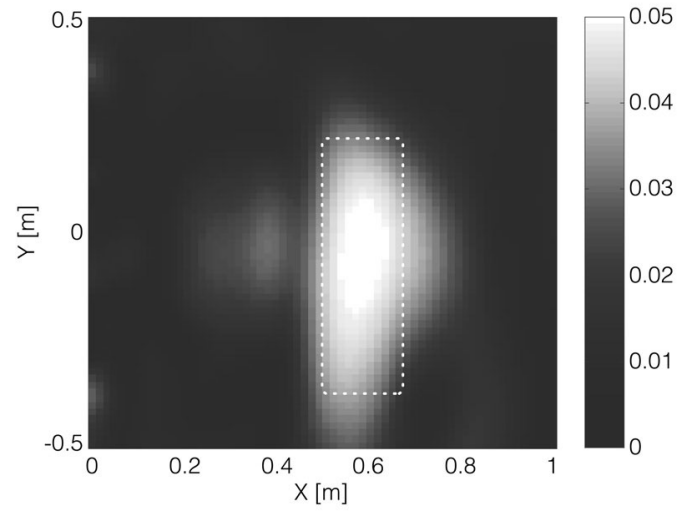

Fig. 8. Case 2: a conductivity anomaly. Modulus of the reconstructed contrast when considering clean data. The reference shape is reported as a contour plot. fig. 9, proves the stability of our approach with respect to noise.

In the last example we show the target is an empty pipe whose cross-section has a ring shape. The pipe has a relative dielectric permittivity of 7 , a conductivity of $1 \times 10^{-3} \mathrm{~S} / \mathrm{m}$ and it is 10 $\mathrm{cm}$ thick. This case is a quite hard one because both $\Delta \varepsilon$ and $\Delta \sigma$ contribute to the scattered field, moreover a large model error $(70 \%)$ is present due to the strong difference between the permittivity of void (inside the pipe) and that of the background, while at the same time a little difference between the pipe and the background is present. In fig. 10 the modulus of the real part of the contrast reconstructed from clean data is plotted. Again, due to the large model error, no quantitative reconstruction is achieved, but the presence of the target as well as the «transition» between the 


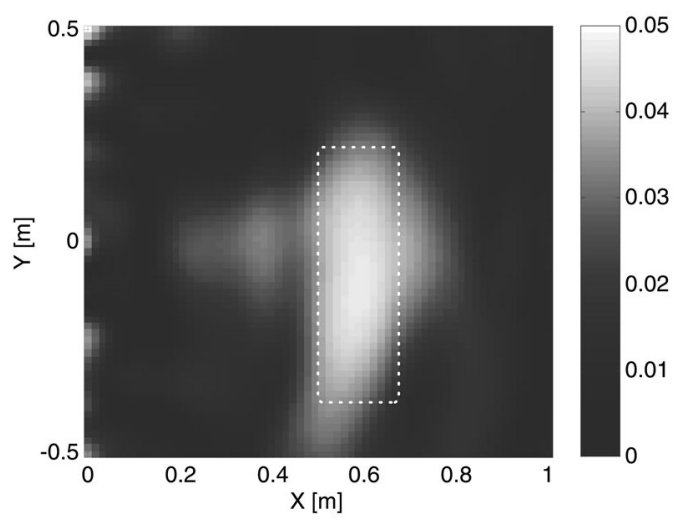

Fig. 9. Case 2: a conductivity anomaly. Modulus of the reconstructed contrast when considering noisecorrupted data $(\mathrm{SNR}=10 \mathrm{~dB})$.

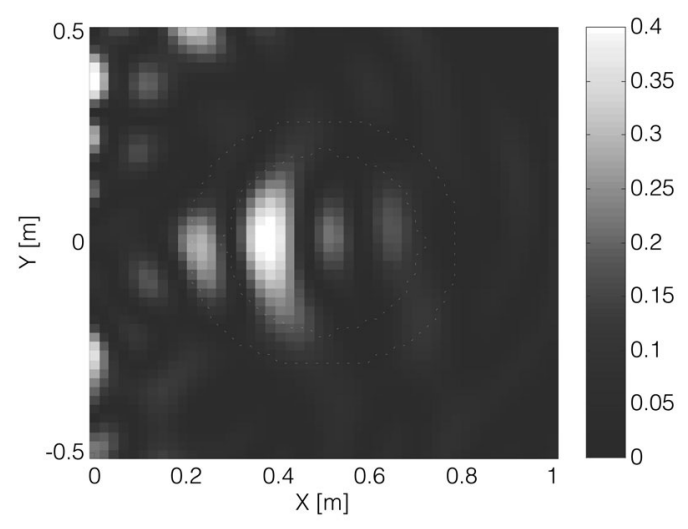

Fig. 11. Case 3: a buried empty pipe. Modulus of the real part of the reconstructed contrast when considering noise-corrupted data $(\mathrm{SNR}=10 \mathrm{~dB})$.

shell and inner part of the pipe can be inferred from the reconstruction. Finally, in fig. 11 the modulus of the real part of the reconstructed contrast when considering noise corrupted data $(\mathrm{SNR}=10 \mathrm{~dB})$ is reported, again proving robustness of the proposed approach against noise.

\section{Conclusions}

A linearized approach elaborated within the framework of the BA for the reconstruction

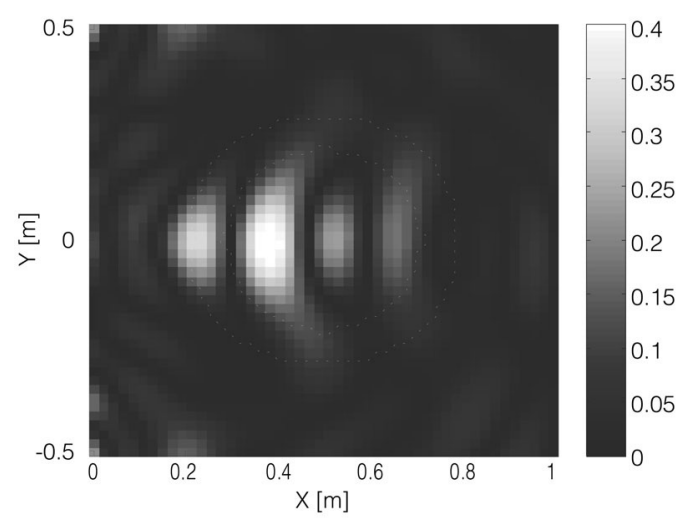

Fig. 10. Case 3: a buried empty pipe. Modulus of the real part of the reconstructed contrast when considering clean data. The reference shape is reported as a contour plot.

of objects embedded in a layered medium has been presented. A suitable formulation of the problem allowed us tackle the dispersive nature of the scattering problem considering a pair of frequency independent unknowns, $\Delta \varepsilon$ and $\Delta \sigma$. This exploits multifrequency data at best.

As shown by several numerical examples, the proposed approach produces an efficient and reliable solution algorithm. In particular, the regularization strategy proves to be very stable since results obtained with «clean» and noisecorrupted data are almost coincident. Moreover, although the assumptions underlying the BA model actually limit the class of retrievable functions (in terms of the permittivity and the extension of the target with respect to that of the background as well as in terms of its spatial variability), the numerical examples show that the approach can provide good (qualitative) results also in cases in which the linear approximation fails.

The SVD tool has been widely exploited in this paper not only to regularize the inverse problem, but also to determine which is the optimal measurement set-up. As a matter of fact, by means of a simple numerical analysis, we were able to determine the suitable number of measurements to perform and the number of working frequencies to consider. 


\section{Appendix.}

The function $g_{21}$ represents the field generated in the first medium at the location $\left(x_{p}, y_{p}\right)$ by a filamentary source located at the point $\left(x^{\prime}, y^{\prime}\right)$ in medium 2 . By relying on the general expression for a layered medium (Chew, 1995) one achieves

$$
\begin{gathered}
g_{21}\left(x_{p}, y_{p}, x^{\prime}, y^{\prime}\right)=\frac{-j}{4 \pi} \int_{-\infty}^{+\infty} \tau_{21} M_{2} \exp \left(j \beta_{1} y_{p}\right) \exp \left[j \kappa\left(x_{p}-x^{\prime}\right)\right] \times\left[\exp \left(-j \beta_{2} y^{\prime}\right)+\right. \\
\left.+R_{23} \exp \left(-j \beta_{2} 2 d\right) \exp \left(j \beta_{2} y^{\prime}\right)\right] d \kappa
\end{gathered}
$$

wherein $\kappa$ is the spatial frequency and

$$
\begin{gathered}
\beta_{i}=\sqrt{\omega^{2} \mu_{0} \varepsilon_{0} \varepsilon_{i}-\kappa^{2}} . \\
\tau_{21}=\frac{2}{\beta_{1}+\beta_{2}}
\end{gathered}
$$

accounts for the transmission between media 1 and 2 for a given spatial frequency,

$$
R_{i j}=\frac{\beta_{i}-\beta_{j}}{\beta_{i}+\beta_{j}} .
$$

is the reflection coefficient between media $i$ and $j$ for a given spatial frequency and

$$
M_{2}=\frac{1}{1-R_{21} R_{23} \exp \left(-j \beta_{2} 2 d\right)}
$$

is an «equivalent» reflection coefficient which takes into account the multiple reflections at the two interfaces.

The function $g_{22}$ represents the field generated in the second medium in the location $(x, y)$ by an elementary source located in the point $\left(x^{\prime}, y^{\prime}\right)$ in the same medium.

Its expression (Chew, 1995) is given by

$$
\begin{gathered}
g_{22}\left(x, y, x^{\prime}, y^{\prime}\right)=\frac{-j}{4} H_{0}^{(2)}\left(\beta_{2} \sqrt{\Delta x^{2}+\Delta y_{-}^{2}}\right)+ \\
-\frac{j}{2 \pi} \int_{-\infty}^{+\infty} \frac{R_{23} M_{2} R_{12}}{\beta_{2}} \exp \left(j \beta_{2} 2 d\right) \cos \left(\beta_{2} \Delta y_{-}\right) \exp (j \kappa \Delta x) d \kappa+ \\
-\frac{j}{4 \pi} \int_{-\infty}^{+\infty} \frac{M_{2}}{\beta_{2}}\left\{R_{21} \exp \left(-j \beta_{2} \Delta y_{+}\right)+R_{23} \exp \left(j \beta_{2} \Delta y_{+}-j \beta_{2} 2 d\right)\right\} \exp (j \kappa \Delta x) d \kappa
\end{gathered}
$$

wherein $H_{0}^{(2)}(\cdot)$ is the zero order second kind Hankel function, $\Delta x=x-x^{\prime}, \Delta y_{-}=y-y^{\prime}$ and $\Delta y_{+}=y+y^{\prime}$. 
By means of the Green-Sommerfeld functions it is also possible to evaluate the incident field in the domain $D$ produced by a filamentary current $I_{0}$ located in the first medium at a distance $y_{t}$ from the interface

$$
\begin{gathered}
E_{\text {inc }}\left(\boldsymbol{r}, \boldsymbol{r}_{t}\right)=-\frac{j \omega \mu_{0} I_{0}}{4 \pi} \int_{-\infty}^{+\infty} \tau_{21} M_{2} \exp \left(j \beta_{1} y_{t}\right) \exp \left[j \kappa\left(x_{t}-x^{\prime}\right)\right] \times \\
\times\left[\exp \left(-j \beta_{2} y^{\prime}\right)+R_{23} \exp \left(-j \beta_{2} 2 d\right) \exp \left(j \beta_{2} y^{\prime}\right)\right] d \kappa .
\end{gathered}
$$

As can be seen, $g_{21}, g_{22}$ and the incident field can be interpreted as 1-dimensional Fourier transforms in the spatial frequency $\kappa$ and therefore can be efficiently computed by means of Fast Fourier Transforms.

Some remarks on the solution of the forward scattering problem can be found in Crocco et al. (2002).

\section{REFERENCES}

BERTERO, M. and P. BoCCACCI (1998): Introduction to Inverse Problems in Imaging, Institute of Physics (Bristol and Philadelphia, U.K.), chapters 5 and 9.

BucCi, O.M., L. CRocco, T. IsERnia and V. PASCAZIO (2001a): Subsurface inverse scattering problems: quantifying qualifying and achieving the available information, IEEE Trans. Geosci. Remote Sensing, 39, 2527-2538.

BuCCI. O.M., N. CARDACE, L. CROCCO and T. ISERniA, (2001b): Degree of non-linearity and a new solution procedure in scalar 2-d inverse scattering problems, $J$. Opt. Soc. Am. A, 18, 1832-1845.

BUCCI, O.M. and T. ISERNIA (1997): Electromagnetic inverse scattering: retrievable information and measurement strategies, Radio Sci., 32, 2123-2138.

CHEW, W.C. (1995): Waves and Fields in Inhomogeneous Media (IEEE Press, New Jersey), 410-414.

Colton, D. and R. KRESS (1992): Inverse Acoustic and Electromagnetic Scattering (Springer Verlag, Berlin).

Crocco, L., R. Persico and F. Soldovieri (2002): A tomographic approach for imaging targets embedded in a layered medium, in Proceedings Ninth International Conference on Ground Penetrating Radar, GPR 2002, Santa Barbara, CA, 353-358.

DANIELS, D.J., (1996): Subsurface Penetrating Radar (IEE Press), chapter 1.

DEming, R.W. and A.J. Devaney (1997): Diffraction tomography for multi-monostatic ground penetrating radar imaging, Inverse Problems, 13, 29-45.

ISERNIA, T., V. PASCAZIO and R. PIERRI (1997): A non-linear estimation method in tomographic imaging, IEEE Trans. Geosci. Remote Sensing, 35, 910-923.
ISERNIA, T., V. PASCAZIO and R. PIERRI (2001): On the local minima in a tomographic imaging technique, IEEE Trans. Geosci. Remote Sensing, 39, 1596-1607.

KLEINMAN, R.E. and P.M. VAN DEN BERG (1993): An extended-range modified gradient technique for profile inversion, Radio Sci., 28, 877-884.

LeOne, G., A. BrancaCcio and R. Pierri (2001): The quadratic distorted approximation for the inverse scattering of dielectric cylinders, J. Optical Soc. Am., Pt. A, 18, 600-609.

PIERRI, R. and G. LEONE (1999): Inverse scattering of dielectric cylinders by a second order Born approximation, IEEE Trans. Geosci. Remote Sensing, 37, 374-382.

Pierri, R., G. LeONE, R. PERSico and F. Soldovieri (2001): Electromagnetic inversion for subsurface applications under the distorted Born approximation, Nuovo Cimento C, 24 (2), 245-261.

Pierri, R., A. BrancaCcio, G. LeONE and F. SOldovieri (2002a): Electromagnetic prospection via homogeneous and inhomogeneous plane waves: the case of an embedded slab, AEÜ, Int. J. Electron. Commun., 56 (1), 11-18.

PIERRI, R., A. LisenO, R. SOLIMENE and F. SOLdOVIERI (2002b): Resolution limits in the Fresnel zone: roles of aperture and frequency, Subsurface Sensing Technologies and Applications, 3 (1), 1-18.

RICHMOND, J.H. (1965): Scattering by a dielectric cylinder of arbitrary cross section shape, IEEE Trans. Antennas Propag., 13, 334-341.

SLANEY, M., A.C. KAK and L.E. LARSEN (1984): Limitations of imaging with first-order diffraction tomography, IEEE Trans. Microwave Theory Tech., MTT-32, 860-874.

WANG, T. and M. ORISTAGLIO (2000): GPR imaging using the generalized Radon transform, Geophysics, $\mathbf{6 5}$, 1553-1559. 